\title{
SESAM - The Society in Europe for Simulation Applied to Medicine
}

The Society in Europe for Simulation Applied to Medicine (SESAM) has now become officially affiliated to the Eur J Anaesthesiol. This move demonstrates the progress of SESAM as a recognised European institution with a developing world-wide impact. The progress of SESAM can be further illustrated by its recent expression of interest for a European research grant in the sixth framework programme (FP6) of the European Commission. This application relates to the establishment of a 'Network of Excellence' across Europe and underlines our need to collaborate more closely as researchers and healthcare providers. Only by assembling our strengths in this way can we show the potential power of simulation and its various related tools. For too long work in this field has been fragmented and not conducted in a systematic manner. The existence of a 'Network of Excellence' would be one method to strengthen the scientific impact of this rapidly developing field by concentrating much of the knowledge and experience which is currently available, but fragmented.

Affiliation with the Eur J Anaesthesiol, an established international journal, demonstrates that SESAM is an acknowledged organisation and gives us a unique opportunity to exchange ideas, concepts and research results. Medicine, in its broadest sense, is progressing at a considerable pace and simulation will soon be transformed into virtual reality in many applications both in and beyond anaesthesia. Research into human factors, so important to our field of performance and improvements in patient safety is a very active field with many results and new methods. It is vital to maintain the pace and to be proactive in all developing areas. The value of the position from which we have started was demonstrated by the number and quality of Abstracts and workshops at the SESAM meeting held in Santander, Spain, in 2002. Never has a meeting been so interdisciplinary and with so many new ideas and concepts. The Abstracts of this meeting appear in this issue of the Journal (pp. 836-850).

Simulation is being accepted by more and more institutions and bodies as a valuable and worthwhile

Correspondence to: Marcus Rall, Center for Patient Safety and Simulation, Department of Anaesthesiology, University Hospital Tübingen, Germany. E-mail: marcus.rall@med.uni-tuebingen.de; Tel: +4907071/2986611

Accepted for publication August 2003 EJA 1506 tool to improve education and training of healthcare professionals. The industry has seen a rise in simulation manufacturers and is more active than ever before. Simulators are available for both adults and children and besides various screen-based software simulators, a new generation of intermediate fidelity simulators has been developed. These new portable simulator systems (e.g. The Emergency Care Simulator ${ }^{\circledR}$ (ECS) from METI and the SimMan ${ }^{\circledR}$ from Laerdal) offer exciting new possibilities for training and research and some of the results of these developments were presented at the recent SESAM meeting in London, UK, in April 2003.

Patient safety has never been more in the focus of medicine than today. Various journals, (e.g. Quality and Safety in Health Care) have covered many of these aspects in the past and others (e.g. the New England Journal of Medicine) have recently started to publish a series on patient safety. The ESA meeting in Lisbon, Portugal, in 2004 will feature a number of lectures on patient safety which have been organized by the Patient Safety Committee of the ESA. Patient safety has been a central theme of study for SESAM members for a considerable time and this growing interest heralds a considerable opportunity for SESAM. The time is clearly right for SESAM to begin to assume a more central role in these developments and to assist the medical community in advancing simulation in medicine. We believe that this affiliation with the Eur $J$ Anaesthesiol is the most appropriate starting point.

M. Rall

SESAM Research Coordinator University Hospital Tübingen, Germany

S. Mönk

SESAM Secretary

University Hospital Mainz, Germany

S. Mather

SESAM Treasurer

Bristol Medical Simulation Centre, UK

A. Rettedal

SESAM Vice-President

Stavanger College, Norway

R. Glavin

SESAM President

Scottish Clinical Simulation Centre

Stirling Royal Infirmary, UK 